\title{
Pulmonic Valve Endocarditis Complicating Streptococcus pneumoniae Community-Acquired Pneumonia
}

\author{
McBath $\mathrm{A}^{1}$, Antony $\mathrm{SJ}^{*}{ }^{1,2}$, Gutierrez $\mathrm{M}^{3}$ and Eisenberg $\mathrm{K}^{4}$ \\ ${ }^{1}$ Paul L. Foster School of Medicine, El Paso, Texas, USA \\ ${ }^{2}$ Center for Infectious Diseases and Travel Medicine, El Paso, Texas, USA \\ ${ }^{3}$ El Paso Cardiology, El Paso, Texas, USA \\ ${ }^{4}$ El Paso Cardiothoracic Specialist, El Paso, Texa, USA
}

${ }^{*}$ Corresponding author: Antony SJ, MD, Center for Infectious Diseases and Travel Medicine, 1205 N Oregon, El Paso, Texas 79902, USA, Fax: (915) 533-4902, Tel: (915)533-4900, E-mail: suresh.antony@att.net

Citation: McBath A, Antony SJ, Gutierrez M, Eisenberg K (2014) Pulmonic Valve Endocarditis Complicating Streptococcus pneumoniae Community-Acquired Pneumonia. J Immunol Infect Dis 1(1): 101. doi: 10.15744/2394-6512.1.101

Received Date: February 20, 2014 Accepted Date: October 01, 2014 Published Date: October 07, 2014

\begin{abstract}
Infective endocarditis is most often seen affecting the left side of the heart, with the pulmonary valve being the least frequently involved valve. The most common organisms responsible for infective endocarditis are Staphylococcus aureus and Streptococcus viridans. Here we report a rare case of pulmonary valve endocarditis due to Streptococcus pneumoniae, a rare pathogen for this setting. The clinical features, presentation, and review of the literature are discussed.
\end{abstract}

\section{Introduction}

Pulmonic valve endocarditis is a rare presentation of community-acquired endocarditis. Usually this occurs in the setting of a previously damaged valve or ventricular septal defect and occasionally a foreign object across the pulmonic valve like a Swan Ganz catheter. The pulmonary valve is the least commonly involved valve associated with infective endocarditis [1]. Endocarditis secondary to Streptococcus pneumoniae is even more infrequent.

We present a patient who initially was diagnosed with community-acquired pneumonia caused by S. pneumoniae with associated bacteremia whose symptoms persisted despite treatment with appropriate IV antibiotics. Subsequent examination, laboratory studies, and imaging ultimately revealed vegetations on the pulmonary valve consistent with infective endocarditis due to $S$. pneumoniae.

\section{Case Report}

We report a 36-year-old Hispanic male who initially presented with complaints of shortness of breath and persistent cough for the previous three months. These symptoms persisted along with a low grade fever. He was admitted to the hospital with a temperature 101.2 ${ }^{\circ} \mathrm{F}$, blood pressure 138/88 $\mathrm{mmHg}$ and a pulse $102 \mathrm{bpm}$. Physical examination revealed a sick looking male with bilateral crackles and rales on auscultation with tachypnea. There were no abnormal heart sounds noted, and the rest of the physical examination was unremarkable. Past medical history was significant for type 2 diabetes mellitus, hypertension, dyslipidemia, and recently diagnosed gastritis with resulting anemia. Labs were significant for hemoglobin $9 \mathrm{~g} / \mathrm{dL}$, hematocrit $27 \%$, and white blood cell count 8,000 cells $/ \mathrm{mm}^{3}$. Blood cultures came back positive for Streptococcus pneumoniae. Admission chest X-ray showed evidence of a multilobar pneumonia involving the lower lobes. He was treated with IV ceftriaxone for 14 days and saw improvement of his fever and cough, although he continued to feel fatigued, tired, and occasional shortness of breath. The patient returned approximately three weeks after completion of the IV antibiotics complaining of abdominal pain, diarrhea, fatigue, and lowgrade fever up to $100^{\circ} \mathrm{F}$. On initial exam, the patient was afebrile with blood pressure $130 / 70 \mathrm{mmHg}$, but otherwise there were no abnormal findings. During the course of this hospital visit, the patient was noted for the first time to have an early systolic murmur. The patient reported he still felt fatigue, tiredness, and occasional shortness of breath, while stating that he just had not felt the same since his initial presentation. Physical exam revealed sinus tachycardia at $100 \mathrm{bpm}$ with a grade I/VI holosystolic murmur at the left upper sternal border. Lab results showed a hemoglobin of $10 \mathrm{~g} / \mathrm{dL}$, hematocrit $30 \%$, and white blood cell count 5,100 cells/ 
$\mathrm{mm}^{3}$. Repeat blood cultures were again positive for Streptococcus pneumoniae. Stool was positive for leukocytosis and negative for C. difficile toxin. Chest X-ray showed persistent infiltrates consistent with prior chest X-rays with new infiltrates in the right upper lobes. Abdominal CT showed evidence of ascending colitis. The patient was started empirically on levofloxacin and metronidazole for presumed infectious colitis. Due to the newly discovered murmur, a 2D surface transthoracic echocardiogram was performed to check for echodensities that would suggest infection of the heart valves; the study revealed a vegetation on the pulmonic valve suggestive of endocarditis. The previous antibiotics were discontinued, and the patient was started again on ceftriaxone as the vegetation was deemed too small for surgery and he had not had any significant thromboembolic phenomena.

Two weeks later, the patient returned for a follow up in the office showing significant improvement with his ongoing antibiotic therapy. However, the next day, he again presented to the hospital with sudden onset coughing, fever, shortness of breath, and chest pain. On physical exam, he was afebrile, blood pressure was 130/70 $\mathrm{mmHg}$, and pulse $78 \mathrm{bpm}$; pleuritic rubs were heard on auscultation of the lungs, and a persistent soft ejection systolic murmur was noted on cardiac exam. Labs were significant for white blood cell count of 14,000 cells/mm $\mathrm{mm}^{3}$, hemoglobin $11 \mathrm{~g} / \mathrm{dL}$, hematocrit 35\%, and glucose $231 \mathrm{mg} / \mathrm{dL}$. Chest CT scan showed right lower lobe infiltrates that were considered evidence of possible pulmonary embolus, at which point septic emboli from the heart valve vegetation was suspected. A transesophageal echocardiogram confirmed the presence of vegetations on the pulmonary valve (Figure 1), at which point the decision was made to consult with cardiothoracic surgery for valve replacement (Figures 2,3,4 \& 5).

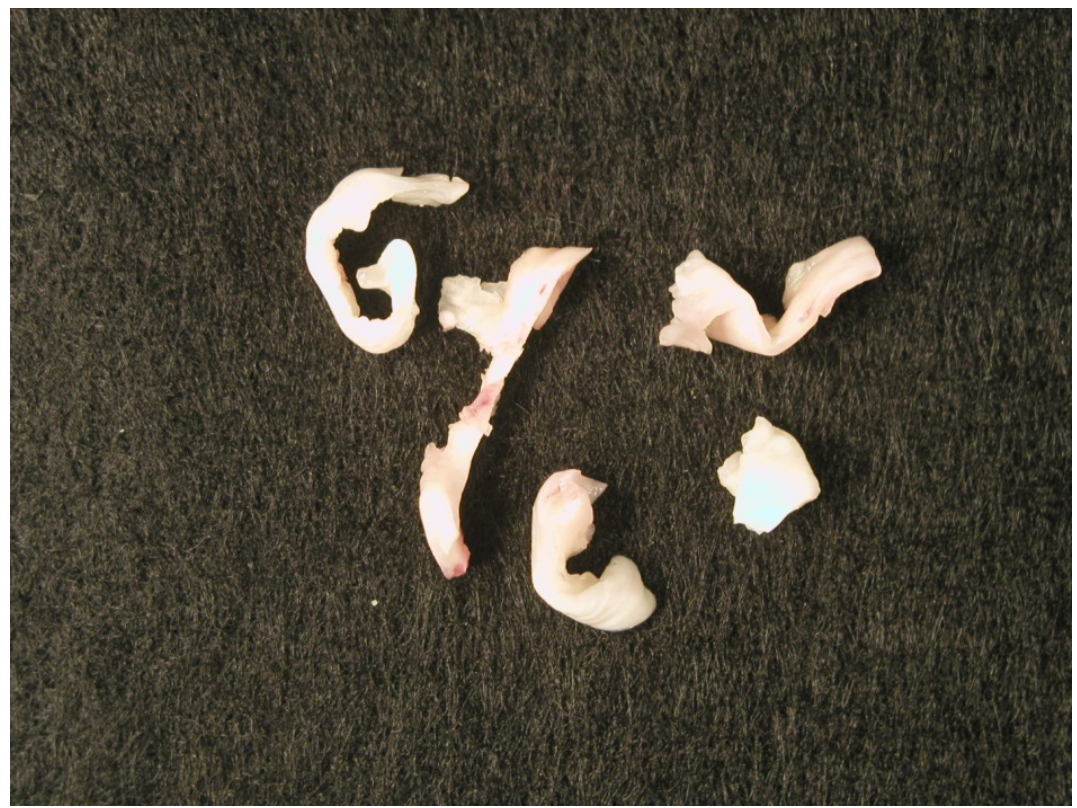

Figure 1: Resected valve with vegetation. Gram stain and cultures revealed streptococcal pneumonia.

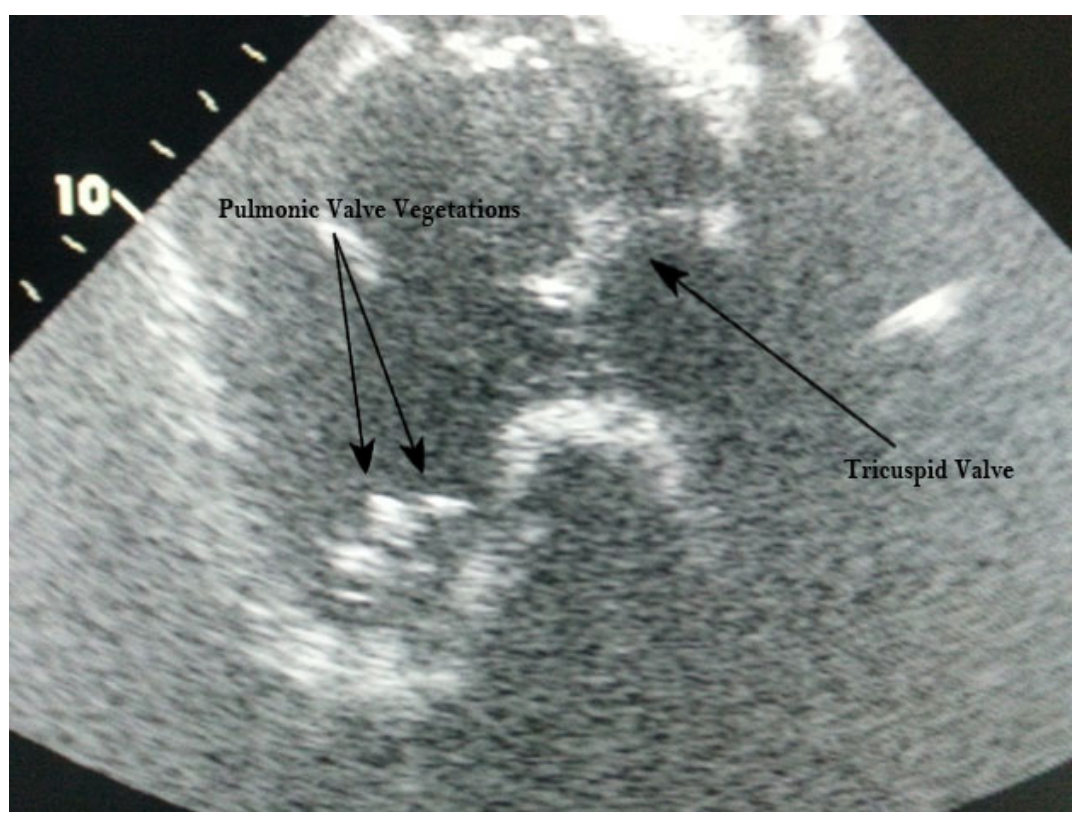

Figure 2: TEE Trans-gastric Right Ventricle Inflow View showing the pulmonic valve vegetation 


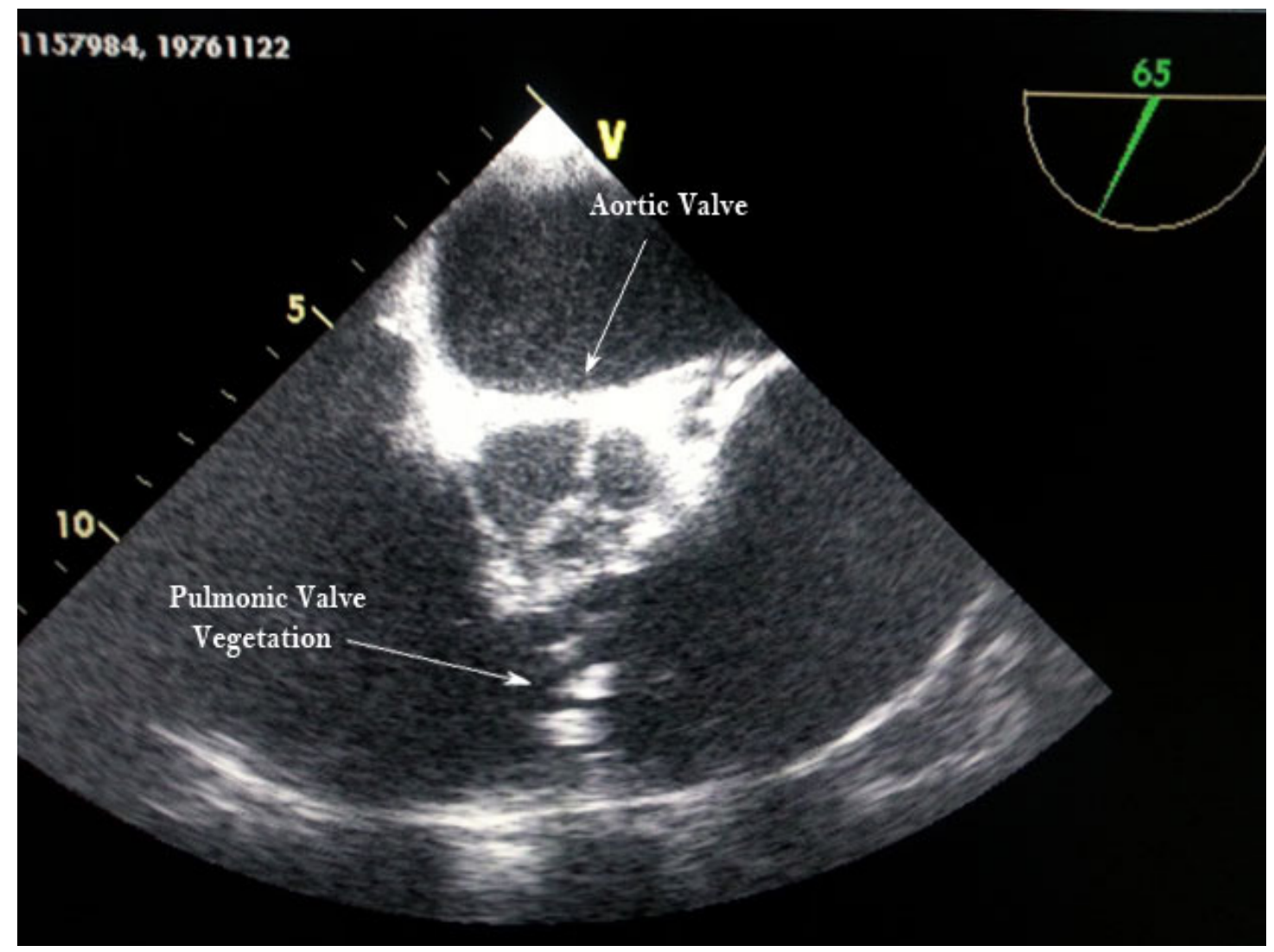

Figure 3 : Mid-esophageal Short-Axis View Showing Pulmonic Valve Vegetation

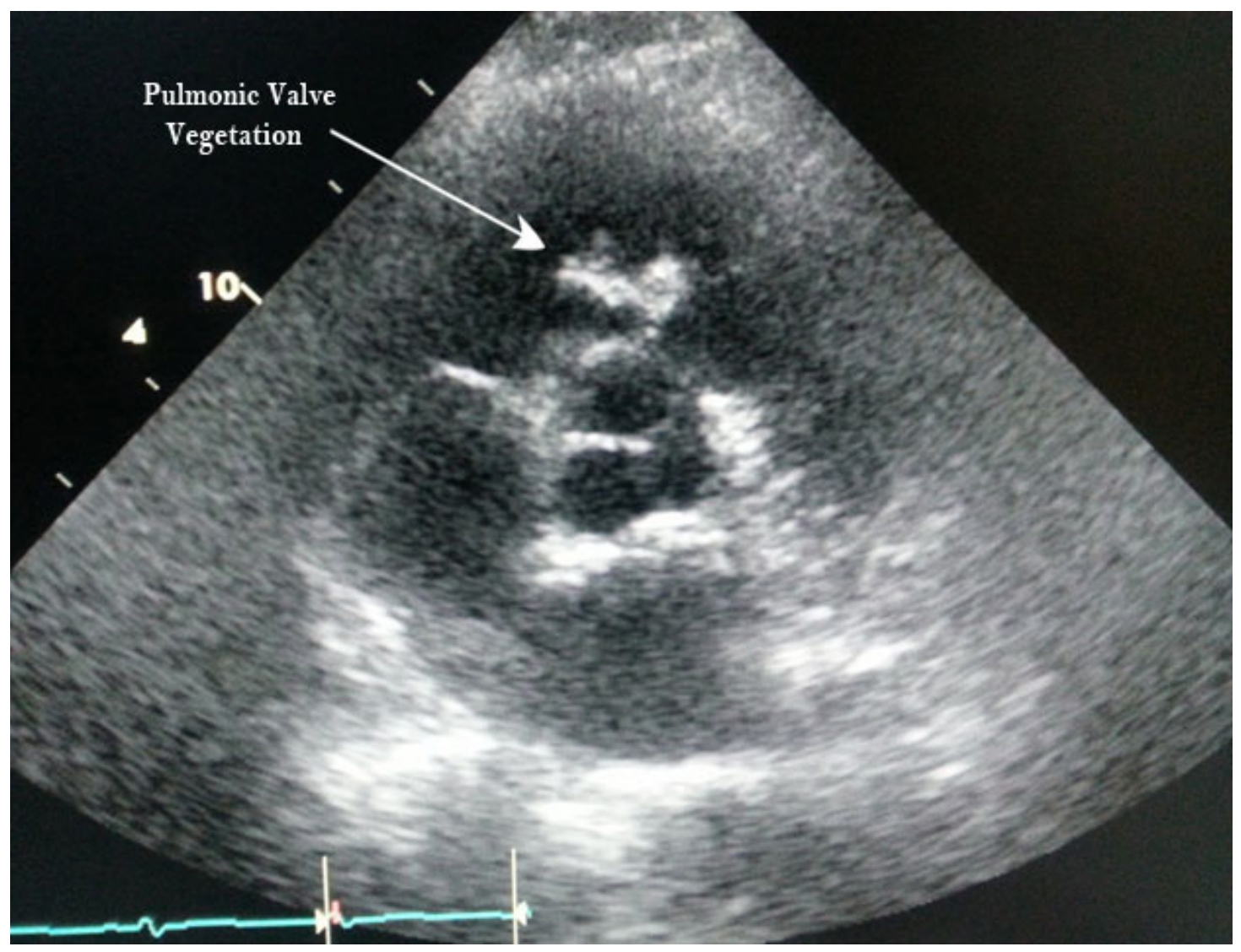

Figure $4: 2 \mathrm{D}$ Surface Transthoracic Echocardiogram. Parasternal Short Axis 


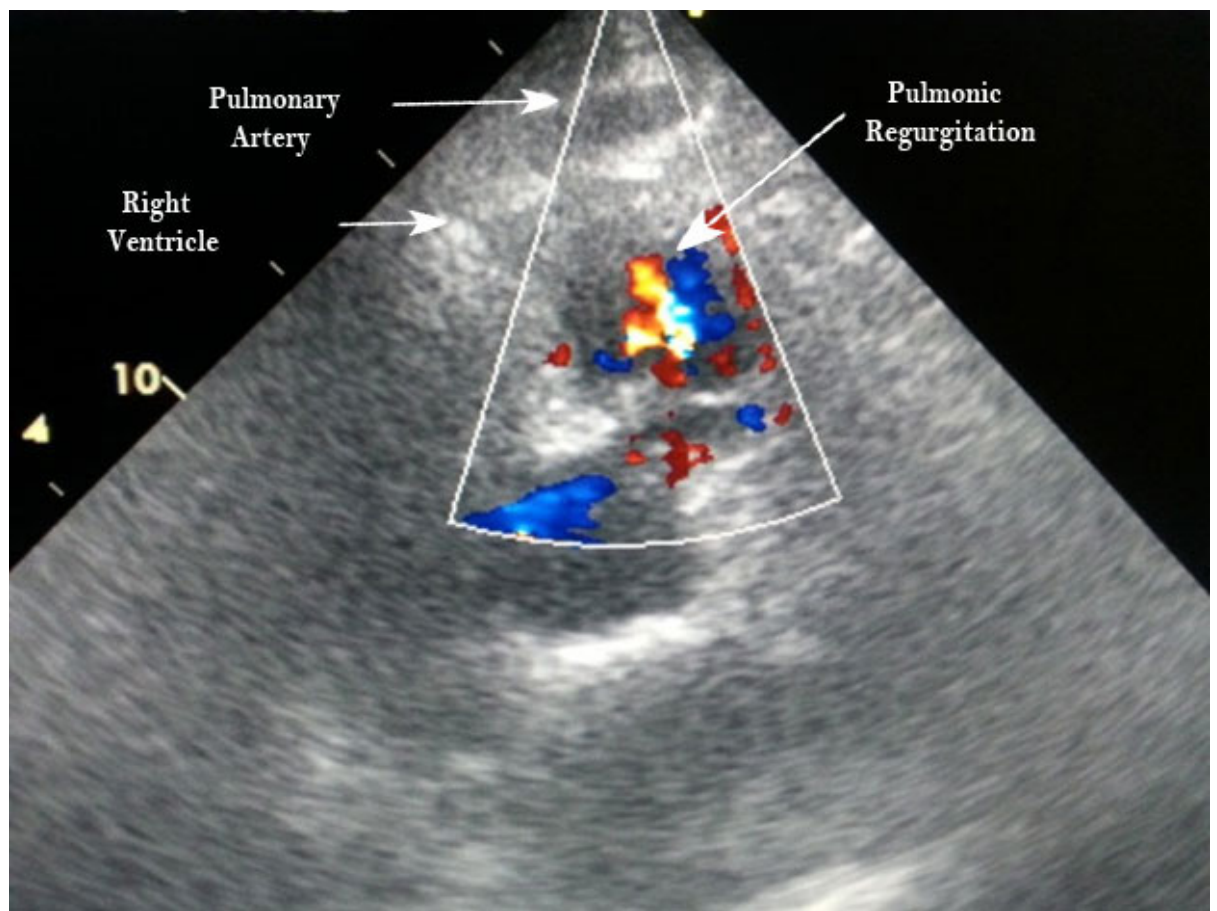

Figure 5: 2D Surface Transthoracic Echocardiogram. Parasternal Short Axis

The patient underwent replacement of the pulmonary valve (Figure 1) due to a lack of response to antibiotic therapy and possible septic emboli involving the lungs. In addition, exploration of the heart revealed a $1 \mathrm{~mm}$ sub-pulmonic ventricular septal defect that had been previously undiagnosed; this defect was determined to be old and well-established rather than a result of the endocarditis. The pulmonary valve was resected and replaced with a \#25 Edwards tissue prosthetic valve, and the septal defect was repaired primarily. The patient's postoperative course was uneventful. He was seen four weeks after his surgery at which time both his pulmonary symptoms and his bacteremia had resolved.

\section{Discussion}

Infective endocarditis is an infection of the inner lining of the heart that usually involves one or more valves and adjacent structures. The vast majority of cases involve the left side of the heart, with only approximately $5 \%$ involving the right side [1]. Multiple valve involvement is also possible (Table 1). Isolated pulmonary valve endocarditis is the rarest form and has been found to occur in less than $1 \%$ of cases [1]. Possible reasoning for its rarity include lower pressure gradients across valves on the right side of the heart compared to the left, lower oxygen content of venous blood, and lower frequency of malformations involving the right side of the heart [2].

\begin{tabular}{|l|l|}
\hline Valve (s) & Incidence (\%) \\
\hline Mitral & $28-45$ \\
\hline Aortic & $5-36$ \\
\hline Tricuspid & $0-6 \%$ \\
\hline Pulmonary & $<1$ \\
\hline
\end{tabular}

Table 1: Incidence of Infective Endocarditis by Valve [1]

Risk factors for infective endocarditis include intravenous drug abuse, alcoholism, sepsis, congenital heart defects or diseases, and central venous catheterization [3]. Of these factors, sepsis and a previously undiagnosed congenital heart defect apply to our patient. Causative organisms can vary depending on each patient's risk factors, but the predominant species involved are Staphylococcus aureus and Streptococcus viridans [2] (Table 2). Definitive diagnosis is via Duke's criteria and requires two major criteria, five minor criteria, or one major + three minor criteria (Table 3) [4].

The causative organism in this case, Streptococcus pneumoniae, is a natural inhabitant of the human nasopharynx. While its presence here is normally benign, spread to another location may be pathogenic; for example, spreading to the lower respiratory tract can lead to pneumonia [6]. Endocarditis caused by S. pneumoniae infection is quite rare and is typically associated with alcohol abuse, which was not seen in our patient [4]. Past reports of S. pneumoniae endocarditis suggest it may manifest as part of the Austrian 
syndrome, consisting of pneumonia, meningitis, and endocarditis [7]. Of these three manifestations, all but meningitis were prominent features of our patient's clinical course. Notably, endocarditis caused by this organism is associated with an extremely high rate of mortality [1].

\begin{tabular}{|l|l|}
\hline Organism & Percent of Cases \\
\hline Staphylococcus aureus & 44.2 \\
\hline Streptococcus spp. & 38.0 \\
\hline Group A & 1.1 \\
\hline Group B & 2.0 \\
\hline Group C & 0.2 \\
\hline Group D & 9.8 \\
\hline S. pneumoniae & 0.6 \\
\hline Other & 24.3 \\
\hline Gram negative rods & 8.6 \\
\hline Other & 9.2 \\
\hline
\end{tabular}

Table 2: Infective Endocarditis by Organism [5]

${ }^{\star}$ Includes infection with multiple organisms

\begin{tabular}{|l|l|}
\hline \multirow{4}{*}{ Major criteria } & Typical organism \\
\cline { 2 - 2 } & Persistent bacteremia (multiple cultures) \\
\cline { 2 - 3 } & Positive serology for Coxiella burnetii \\
\cline { 2 - 2 } & Positive echo results for vegetations or abscess \\
\hline \multirow{4}{*}{ Minor criteria } & Valvular heart disease \\
\cline { 2 - 2 } & History of IV drug abuse \\
\cline { 2 - 2 } & Fever higher than $38^{\circ} \mathrm{C}$ \\
\cline { 2 - 2 } & Vasculitis, skin lesions \\
\cline { 2 - 2 } & Suggestive (but not definitive) echo results \\
\cline { 2 - 2 } & Single positive blood culture \\
\hline
\end{tabular}

Table 3: Duke’s criteria for diagnosis of infective endocarditis [4]

Isolated pulmonary valve endocarditis is frequently associated with respiratory symptoms due to the anatomical and functional proximity of the pulmonary valve to the lungs. Embolization of septic vegetations to the lung, in particular, is an important complication that should be kept in mind in patients with shortness of breath and other respiratory symptoms [8]. Indeed, it is possible our patient's shortness of breath and cough were consequences of septic emboli from the pulmonary valve lodging in the pulmonary vasculature. In addition, it is possible a septic embolus was the cause of the patient's pneumonia rather than the pneumonia being the precipitating event that ultimately led to his endocarditis [9]. Conversely, the presence of a congenital ventricular septal defect could have given blood returning from the lungs open access to the right side of the heart, allowing pulmonary bacteria to infect the pulmonic valve [10].

In the majority of cases, right heart endocarditis progresses with a benign course relative to infections involving the left heart. While surgical treatment of mitral or aortic valve endocarditis is more common, right heart endocarditis is most often successfully treated with medication alone [1,11]. In more serious cases, surgery may also be utilized for endocarditis involving the right heart valves. Generally accepted indications for surgery include infection with certain organisms (for instance, Staphylococcus aureus or Gram-negative bacilli), secondary heart failure, lack of response to antibiotic therapy, severe valve dysfunction, pulmonary involvement such as septic emboli or cavitation, and systemic involvement such as disseminated intravascular coagulation or acute renal failure $[10,12]$. In our case, the indications for surgery were the lack of response to antibiotic therapy in addition to pulmonary involvement.

In summary, the case presented demonstrates an unusual case of isolated pulmonary valve endocarditis with Streptococcus pneumoniae, an organism not often associated with endocarditis. The case suggests that pulmonary valve endocarditis should be considered in patients who present with recurrent pulmonary symptoms, especially if there is high suspicion based on associated symptoms such as recurrent fevers or persistently positive blood cultures despite treatment, as seen in this patient. Notably, these symptoms can mimic pneumonia, so appropriate diagnostic tests should be performed to distinguish true pneumonia from a pneumonia-like presentation secondary to embolization from pulmonary valve endocarditis. 


\section{References}

1. Smith S, Rivera J, Antony S (2009) Use of Daptomycin in the Treatment of Prosthetic Pulmonary Valve Endocarditis. The Internet Journal of Infectious Diseases 8: 1 .

2. Moreira D, Correia E, Rodrigues B, Santos L, Capelo J, et al. (2012) Isolated pulmonary valve endocarditis in a normal heart. Rev Port Cardiol 31 : 615-7.

3. Sá MI, Môço R, Cabral S, Reis AH, Pereira LS, et al. (2007) Isolated pulmonary valve endocarditis due to Pseudomonas aeruginosa. Rev Port Cardiol 26: 43-8.

4. Keys TF (2010) Infective Endocarditis. Disease Management Project, Cleveland Clinic Center for Continuing Education, Cleveland, United States.

5. Bor DH, Woolhandler S, Nardin R, Brusch J, Himmelstein DU (2013) Infective endocarditis in the U.S., 1998-2009: a nationwide study. PLoS One 8: e60033.

6. Donkor ES (2013) Understanding the pneumococcus: transmission and evolution. Front Cell Infect Microbiol 3: 7.

7. Kanakadandi V, Annapureddy N, Agarwal SK, Sabharwal MS, Ammakkanavar N, et al. (2013) The Austrian syndrome: a case report and review of the literature. Infection 41: 695-700.

8. Bilen E, Yasar AS, Bilge M, Kurt M, Karakas F, et al. (2008) Isolated pulmonic valve endocarditis in an adult patient with ventricular septal defect and infundibular pulmonary stenosis. Echocardiography 25: 904-7.

9. Bhavani SS, Slisatkorn W, Rehm SJ, Pettersson GB (2006) Deep sternal wire infection resulting in severe pulmonary valve endocarditis. Ann Thorac Surg 82: 1111-3.

10. Park HE, Cho GY, Kim HK, Kim YJ, Sohn DW (2009) Pulmonary valve endocarditis with septic pulmonary thromboembolism in a patient with ventricular septal defect. J Cardiovasc Ultrasound 17: 138-40.

11. Deng H, Ma Y, Zhai H, Miao Q (2013) Surgical valve repair of isolated pulmonary valve endocarditis. Interact Cardiovasc Thorac Surg 16: $384-6$.

12. Abdelbar A, Azzam R, Yap KH, Abousteit A (2013) Isolated pulmonary infective endocarditis with septic pulmonary embolism complicating a right ventricular outflow tract obstruction: scarce and devious presentation. Case Rep Surg 2013: 746589.

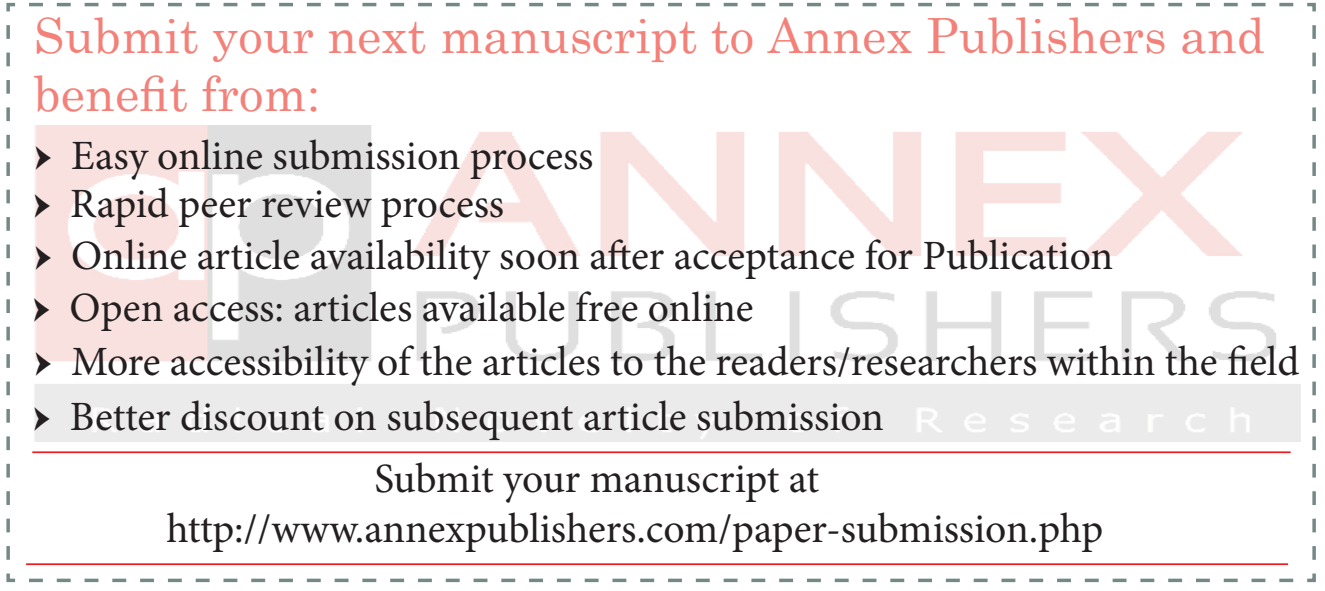

